Betreuung von Tumorpatienten

\title{
Neues Internetportal für onkologische Pflegekräfte: www.pflege-onkologie.de
}

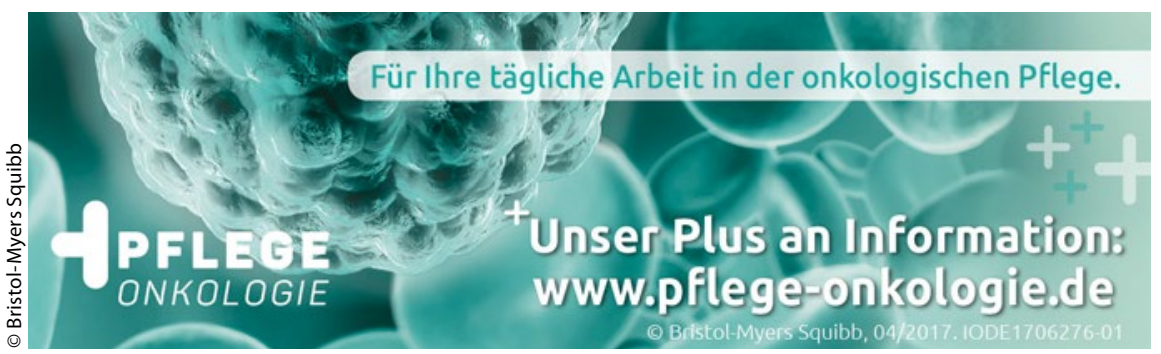

Mit der Immunonkologie konnte das herkömmliche und etablierte Spektrum der Tumorbehandlungen Operation, Bestrahlung, Chemo- und zielgerichtete Therapien in jüngster Zeit um eine weitere effektive Option erweitert werden (Borghaei $\mathrm{H}$ et al.; Eur J Pharmacol 2009; 625: 41-54). Immunonkologische Konzepte nutzen die natürliche Fähigkeit des körpereigenen Abwehrsystems, Tumore zu erkennen, zu bekämpfen und zu zerstören. Wie bei den anderen Behandlungsmaßnahmen auch, ist vor Beginn einer immunonkologischen Therapie ein ausführliches Patientengespräch unverzichtbar, um über die Möglichkeiten dieser
Strategie, eventuelle Nebenwirkungen und deren Management aufzuklären.

Im oftmals hektischen Klinikalltag haben Pflegekräfte häufig einen intensiveren Kontakt zum Patienten als Ärzte und sind für viele Probleme oft die ersten Ansprechpartner. Um das Pflegepersonal bei dieser anspruchsvollen Aufgabe zu unterstützen, bietet das Unternehmen Bristol-Myers Squibb das speziell für Pflegekräfte entwickelte, neue Internet-Portal www.pflegeonkologie.de an. Die Website greift ein breites Spektrum an Themen auf, die im pflegerischen Alltag wichtig sind. So liefert sie Informationen zu mehr als 20 Tumorin- dikationen und zu den verschiedenen Behandlungsoptionen in der Krebsmedizin. Nebenwirkungen und Supportivmedizin, Möglichkeiten der psychoonkologischen Unterstützung und die bei Tumorpatienten wichtige Ernährung werden angesprochen. Außerdem informiert die Website mit einem Kongresskalender über Fort- und Weiterbildungsangebote im pflegerischen Bereich. Abgerundet wird die Seite durch weiter führende Informationsangebote, wie Buchtipps, Fachzeitschriften und ein Glossar medizinischer Fachbegriffe. Schließlich stehen Download-Materialien, wie Ernährungstipps für Patienten während einer (immun)onkologischen Therapie zur Verfügung. Die Website ist frei zugänglich; eine Registrierung ist nicht erforderlich und kann von jedem Endgerät, wie Desktop, Tablet oder Smartphone aufgerufen werden. Mit diesem neuen Angebot setzt Bristol-Myers Squibb sein lange bestehendes Engagement für die Fortbildung von Pflegekräften fort. Bereits in der Vergangenheit war das forschende Pharmaunternehmen auf diesem Gebiet sehr aktiv und mit Ständen und Symposien auf Veranstaltungen für Pflegekräfte vertreten. Auch zukünftig wird Bristol-Myers Squibb, als Partner für die Pflege, sein Engagement fortsetzen. (reck)

\section{Supportive Onkologie Gegen Mukositis}

— In der Pipeline von Mundipharma im Bereich Supportive Care in der Onkologie befindet sich durch eine gebrauchsfertige Mundspüllösung zur Behandlung der oralen Mukositis bei Tumortherapie (Ectomusal ${ }^{\circledR}$ ). Das Medizinprodukt enthält Ectoin ${ }^{\circledR}$, das ein Bestandteil extremophiler Bakterien (z. B. Halomonos elongata) ist und es diesen ermöglicht, in extremem Milieu zu überleben. Ectoin hat durch die Ausbildung stabiler Wasserstoffbrückenbindungen einen hydratisierenden Effekt. Die Stabilisierung von Zellmembranen stärkt die Barrierefunktion und reduziert Entzündungen.

Einführungspressekonferenz ${ }_{\text {"Cysel }}{ }^{\circledR}$ - anorganisches Selen bei nachgewiesenem Selenmangel und Ectomusal ${ }^{\oplus}$ - Mundspüllösung gegen orale Mukositis bei Tumorpatienten", DGHO-Kongress 2017, Stuttgart, 30. September 2017 (Veranstalter: Mundipharma Deutschland GmbH \& Co.KG)

\section{Alte Patienten \\ Schwindel ist meist multikausal}

— Schwindel ist eines der häufigsten Symptome bei älteren Menschen und ein Risikoindikator für Stürze, Synkopen, Pflegebedürftigkeit und ein erhöhtes Schlaganfallrisiko. Die Anamnese bei älteren Patienten sollte Art, Auslöser, Begleitsymptome und die Frage nach Bewusstseinsverlust ebenso einschließen wie die genaue Medikation inklusive der OTC-Medikamente. Auch indiziert ist die körperliche neurologische Untersuchung. Therapeutische Ansatzpunkte sind zunächst die Suche und Behandlung eventuell vorhandener monokausaler Schwindelursachen, so der Geriater Professor Karl-Günter Gaßmann. Indiziert und oft sehr erfolgreich bei gemischtem und multifaktoriellem Schwindel seien das Training von Gangsicherheit, Gleichgewicht, Kraft sowie ein Verhaltenstraining, das sich auch der Bewältigung der Angst vor den Stürzen widme. Nimmt der Patient schwindelindu- zierende Medikamente wie Sedativa, zentral wirkende Analgetika, Antihypertensiva, Spasmolytika oder Parkinsonmedikamente, sollte eine Umstellung oder ein Auslassversuch mit nachfolgendem Ausschleichen probiert werden. Die medikamentöse Schwindeltherapie greife bei gewisser Indikation, sollte aber keine Dauertherapie sein. Als einziges natürliches Präparat wird Vertigoheel ${ }^{\circledR}$ bei akutem Schwindel seit einem Jahr in der hausärztlichen DEGAM-Leitlinie empfohlen. Wegen des Fehlens von Nebenund Wechselwirkungen eignet es sich vor allem für multimorbide ältere Patienten und wird von vielen GKV als freiwillige Satzungsleistung erstattet.

(hae)

Lunchsymposium „Schwindel - eine interdisziplinäre Herausforderung", Jahrestagung der Deutschen Gesellschaft für Geriatrie (DGG), Frankfurt am Main, 29. September 2017 (Veranstalter: Heel) 